\title{
Mini-Tn7 vectors for stable expression of diguanylate cyclase PleD* in Gram-negative bacteria
}

\author{
Lorena Romero-Jiménez, David Rodríguez-Carbonell, María Trinidad Gallegos, Juan Sanjuán \\ and Daniel Pérez-Mendoza* (1)
}

\begin{abstract}
Background: The cyclic diguanylate (c-di-GMP) is currently considered an ubiquitous second messenger in bacteria that influences a wide range of cellular processes. One of the methodological approaches to unravel c-di-GMP regulatory networks involves raising the c-di-GMP intracellular levels, e.g. by expressing a diguanylate cyclase (DGC), to provoke phenotypic changes.

Results: We have constructed mini-Tn7 delivery vectors for the integration and stable expression of the pleD* gene encoding a highly active DGC, which can be used to artificially increase the intracellular levels of c-di-GMP in Gram negative bacteria. The functionality of these new vectors has been validated in several plant-interacting a- and $\gamma^{-}$ proteobacteria. Similarly to vector plasmid-borne pleD*, the genome-borne mini-Tn7pleD* constructs provide significant increases in intracellular c-di-GMP, provoking expected phenotypic changes such as enhanced polysaccharide production, biofilm formation and reduced motility. However, the mini-Tn7pleD* constructs resulted far more stable in the absence of antibiotics than the plasmid-based pleD* constructs. Furthermore, we have also implemented an inducible system to modulate pleD* expression and intracellular c-di-GMP rises "on demand".
\end{abstract}

Conclusions: mini-Tn7pleD* constructs are very stable and are maintained during bacterial free-living growth as well as during interaction with eukaryotic hosts, in the absence of selective pressure. This high stability ensures experimental homogeneity in time and space with regard to enhancing c-di-GMP intracellular levels in bacteria of interest.

Keywords: c-di-GMP, mini-Tn7, Signal transduction, Biofilms, Exopolysaccharide production, Bacterial motility, Plasmid stability

\section{Background}

The cyclic diguanylate (c-di-GMP) was discovered only 27 years ago as an allosteric activator of bacterial cellulose synthase, but is currently considered an ubiquitous second messenger in bacteria that influences a wide range of cellular processes, including flagellum-mediated motility, cell cycle and exopolysaccharide (EPS) biosynthesis, as well as bacterial virulence [1]. c-di-GMP signalling systems are generally composed of three major constituents: diguanylate cyclases (DGCs, synthesize c-di-GMP from two GTP molecules), phosphodiesterases (PDEs,

\footnotetext{
* Correspondence: dpmendoza@eez.csic.es

Departamento Microbiología del Suelo y Sistemas Simbióticos, Estación Experimental del Zaidín, Consejo Superior de Investigaciones Científicas (CSIC), Granada, Spain
}

(c) 2015 Romero-Jiménez et al. Open Access This article is distributed under the terms of the Creative Commons Attribution 4.0 International License (http://creativecommons.org/licenses/by/4.0/), which permits unrestricted use, distribution, and reproduction in any medium, provided you give appropriate credit to the original author(s) and the source, provide a link to the Creative Commons license, and indicate if changes were made. The Creative Commons Public Domain Dedication waiver (http://creativecommons.org/publicdomain/zero/1.0/) applies to the data made available in this article, unless otherwise stated.
In general, the GGDEF domain of DGCs and the EAL or HD-GYP domains of PDEs are responsible for DGC and PDE activities, respectively, and balanced control of these opposite activities determines c-di-GMP homeostasis within the cell [3]. Genome analyses have revealed that the number of proteins with DGC and PDE domains is variable, highlighting the ability of bacteria to adapt to different habitats, the range of environmental stimuli perceived and/or the cellular functions affected by them. For instance, free-living bacteria with complex environmental lifestyles and co-evolutionary relationships with eukaryotes possess far more c-di-GMP-metabolizing enzymes than obligate parasites $[1,4,5]$. In that sense, cellular levels of c-di-GMP can be viewed as integral outputs of 
bacterial sensory systems that perceive various biotic and abiotic conditions. c-di-GMP translates input signals into the modulation of cellular behaviours by binding to diverse effector molecules, which so far include specific c-di-GMP receptor proteins (with PilZ, GIL or degenerate GGDEF/ EAL domains), c-di-GMP-binding transcription factors, and RNA motifs (riboswitches) [2, 3, 6-9]. The large diversity of effector elements is indicative of the c-di-GMP regulation at multiple levels: transcriptional, posttranscriptional and posttranslational [1, 2, 10-12]. However, the multiplicity of DGCs and PDEs contrast with the comparatively few albeit functionally diverse c-di-GMP receptors/effectors identified so far, suggesting the existence of yet many unknown effectors. Thus, additional approaches besides genomics and bioinformatics need to be implemented to uncover novel c-di-GMP regulation pathways and targets, particularly in bacteria with complex lifestyles.

One such approach involves artificial modification of the c-di-GMP economy, by either overexpressing a DGC or a PDE, to identify associated phenotypic changes. In a recent work, the c-di-GMP levels of several plantinteracting bacteria were increased by expressing the DGC PleD* [13]. The pleD* gene expressed from a plasmid vector $(\mathrm{pJBpleD} *)$ altered a number of free-living phenotypes, as well as the interaction with their plant hosts. pleD" overexpression has also proven to be useful for uncovering novel and otherwise cryptic EPSs in different bacteria $[14,15]$. Although the pleD* plasmid was certainly a powerful tool, its use was limited by its low stability under non selective conditions (i.e., absence of antibiotics), which often led to rapid loss, particularly in association with plants [13]. To overcome this limitation, we have constructed new vehicles based on the $\operatorname{Tn} 7$ transposon $[16,17]$ for genome integration of the $p l e D^{*}$ gene. $\operatorname{Tn} 7$ inserts into a specific site called $a t t \operatorname{Tn} 7$ and with a determined orientation [18, 19]. Most bacteria possess a single att $\operatorname{Tn} 7$ site [20-22], which is frequently localized downstream of the $\mathrm{glm} S$ gene (encoding glucosamine-fructose-6-phosphate aminotransferase), and where transposon insertions do not usually affect bacterial fitness. Indeed, $\operatorname{Tn} 7$ transposon derivatives have been widely used to introduce genes into bacterial chromosomes and insertions of Tn7 transposon have been successfully obtained in a plethora of different bacteria [16]. Stability and efficacy of the pleD* mini-transposons have been tested in plant-interacting bacteria of the genera Pseudomonas, Rhizobium and Sinorhizobium. In addition, an inducible system was also developed to modulate $p l e D^{*}$ expression and intracellular c-di-GMP rises on demand.

\section{Methods}

\section{Bacteria and culture conditions}

Bacteria and plasmids used in this work are listed in Additional file 1: Table S1. E. coli and Pseudomonas strains were grown routinely in Luria-Bertani broth (LB; containing $10 \mathrm{~g} / \mathrm{L}$ tryptone, $5 \mathrm{~g} / \mathrm{L}$ yeast extract, $5 \mathrm{~g} / \mathrm{L}$ $\mathrm{NaCl})$ at $37{ }^{\circ} \mathrm{C}$ or $28{ }^{\circ} \mathrm{C}$ respectively. Cultures of rizobial strains (Sme, Ret and Rle) were grown at $28{ }^{\circ} \mathrm{C}$ in TY broth (tryptone-yeast extract- $\mathrm{CaCl}_{2}$ ) [23] for Sme and Ret and YGT broth (glucose $15 \mathrm{~g} / \mathrm{L}$, tryptone $5 \mathrm{~g} / \mathrm{L}$, $\mathrm{CaCl}_{2} \cdot 2 \mathrm{H}_{2} \mathrm{O} 0.6 \mathrm{~g} / \mathrm{L}$, yeast extract $2.5 \mathrm{~g} / \mathrm{L}$ ) for Rle. MM medium [24] was used for both rhizobial strains and Pto in different assays. When required, antibiotics were added at the following final concentrations: Tetracycline (Tc), $10 \mu \mathrm{g} / \mathrm{ml}$ for E. coli, Pto and Sme and $5 \mu \mathrm{g} / \mathrm{ml}$ for Ret and Rle; Kanamycin $(\mathrm{Km}) 50 \mu \mathrm{g} / \mathrm{ml}$ for all strains. All free-living cultures of strains carrying pJB3Tc19 or pJBpleD* plasmids contained Tc to prevent plasmid losses, except to evaluate the loss of plasmids without antibiotic pressure.

Stability of all mini-Tn7 constructs was evaluated in all strains. Overnight cultures grown under Tc or Km selection were diluted 1/100 in nonselective LB (Pto), TY (Sme and Ret) or YGT (Rle) media, and incubated for $24 \mathrm{~h}$ at $28^{\circ} \mathrm{C}$ with shaking. Several rounds of dilutions in nonselective media were repeated for at least 100 generations. After this, serial dilutions were spread on nonselective and selective agar plates, and CFUs (colony forming units) counted after incubation at $28^{\circ} \mathrm{C}$. Marker stability was determined as the ratio (\%) of CFUs grown in selective medium out of the total CFUs appeared in nonselective plates.

\section{Construction and insertion of mini-Tn7 vectors into Gram-negative bacteria}

The gene $p l e D^{*}$ together with the lac promoter was PCR amplified from pJBpleD* vector [13] with pJB3Tc19-F and pleDTn7 primers. The fragment was cloned in $\mathrm{pCR}^{\circ}{ }^{-} \mathrm{XL}-$ $\mathrm{TOPO}^{\circ}$ and the resulting vector pTOPO-pleD* was $^{*}$ digested with EcoRI and SacI. The insert was subcloned in pUC18T-mini-Tn7T (AY599230; [16] previously digested to give plasmid mini-Tn7pleD*. Kanamycin $\left(\mathrm{Km}^{\mathrm{r}}\right)$ or Tetracycline $\left(\mathrm{Tc}^{\mathrm{r}}\right)$ resistance cassettes from $\mathrm{p} 34 \mathrm{~S}-\mathrm{Km}$ (AF062080) and p34S-Tc (AF062082), respectively, were introduced, after KpnI digestion, adjacent to the pleD* gene, obtaining mini-Tn7pleD*Km and mini-Tn7pleD*Tc, respectively. To obtain control strains without the gene pleD*, a NcoI internal deletion of 1114 bp of the $1380 \mathrm{bp}$ of ple $D^{*}$ was performed, resulting in plasmids miniTn7Km and mini-Tn7Tc. Mini-Tn7 plasmids containing the pleD $^{*}$ gene were maintained in E. coli $\beta 2155\left(\right.$ lacl $\left.^{q}\right)$ [25] to prevent pleD* overexpression, whereas control plasmids with mini-Tn7Km and Tc plasmids were maintained in E. coli $\beta 2163$ strain [25].

Triparental matings, as described in [26] were employed to deliver the mini- $\operatorname{Tn} 7$ constructs into the genomes of Pseudomonas syringae pv. tomato DC3000 (Pto), Sinorhizobium meliloti 8530 (Sme), Rhizobium etli CFN42 (Ret) and Rhizobium leguminosarum bv. viciae 
UPM791 (Rle). E. coli $\beta 2163$ bearing the pUX-BF13 plasmid carrying the transposase genes was used as helper strain for transposition.

\section{Motility assays}

Motility assays were carried out as described in [13]. For swimming motility the strains were resuspended from MM plates and adjusted to an $\mathrm{OD}_{600}$ of 1 . Two $\mu \mathrm{l}$ were spotted onto semisolid Bromfield medium (0.3\% agar) and halo diameter measured after incubation at $28{ }^{\circ} \mathrm{C}$. Surface motility was analysed using a protocol previously described [27]. We used semisolid MM plates containing $0.6 \%$ purified agar (Agar Noble, Difco), and a representative migration zone from one of the three biological replicates for each strain were imaged after $24-48 \mathrm{~h}$ at $28{ }^{\circ} \mathrm{C}$ for Pto, and $72 \mathrm{~h}$ at $28{ }^{\circ} \mathrm{C}$ for Ret and Rle.

\section{Congo red and calcofluor binding assays}

To observe the production of exopolysaccharides, Sme, Ret and Pto strains were grown on solid MM plates supplemented with Congo red (CR; $125 \mu \mathrm{g} / \mathrm{ml})$ or with calcofluor (CF; $200 \mu \mathrm{g} / \mathrm{ml}$ ). Rle strains were grown on YGT media with the same concentration of CR and CF described above. Calcofluor binding was observed under UV light. CR and CF plates were photographed after 3 days incubation at $28{ }^{\circ} \mathrm{C}$.

To quantify CF binding, $500 \mu \mathrm{l}$ of a starting culture in rich broth was washed twice with $\mathrm{MM}$ and diluted $1 / 100$ into $10 \mathrm{ml}$ flasks containing $\mathrm{MM}$ supplemented with CF $(100 \mu \mathrm{M})$. Flasks were incubated for $48 \mathrm{~h}$ at $28{ }^{\circ} \mathrm{C}\left(24 \mathrm{~h}\right.$ at $20{ }^{\circ} \mathrm{C}$ for Pto). Afterwards, cultures were centrifuged and supernatants removed. The pellets were suspended in $2 \mathrm{ml}$ distilled water and disposed in 24-well plates. Measures of three replicates from independent cultures for each strain were performed in a PTI fluorimeter (Photon Technology International).

\section{Biofilm assays}

All strains were resuspended from a MM plate, washed with $\mathrm{MM}$ and diluted to a $\mathrm{DO}_{600}$ of 0.1. Aliquots of $200 \mu \mathrm{l}$ were placed into the wells of sterile 96-well polystyrene plates (Sarstedt) and left in a humid chamber at $28{ }^{\circ} \mathrm{C}$ for 3 days. After incubation, the liquid from the wells was removed by aspiration and wells were washed with $240 \mu \mathrm{l}$ of deionised water. $240 \mu \mathrm{l}$ of Crystal Violet (CV; $0.1 \%$ in water) was added to each well and left to stain for $1 \mathrm{~h}$. The excess of crystal violet was removed by aspiration and each well was washed carefully with $240 \mu \mathrm{l}$ of deionised water three times. $240 \mu \mathrm{l}$ of $70 \%$ ethanol was added to each well and the plate was gently agitated for at least $1 \mathrm{~h}$. Ethanol suspension was diluted $1 / 2$ for Ret and $1 / 7$ for Rle for purple color quantification. Eight technical replicates from three separate cultures for each strain were measured at A550 nm in a Sunrise microplate reader (Tecan).

\section{Intracellular c-di-GMP measurements}

c-di-GMP was extracted using a protocol described in [13]. Bacteria were grown in $10 \mathrm{ml}$ of TY for Ret and Sme, YGT for Rle or LB broth for Pto. The area of the ion $\mathrm{m} / \mathrm{z} 540$ peak was used to estimate the amount of c-di-GMP in each sample. For quantification, a standard curve was established using synthetic c-di-GMP (Axxora) dissolved in ammonium acetate $(10 \mathrm{mM} \mathrm{pH} 5.5)$ at a range of concentrations $(20 \mathrm{nM}, 200 \mathrm{nM}, 2 \mu \mathrm{M}$ and $20 \mu \mathrm{M})$. After subtracting the basal $250 \mathrm{nM}$ spike, c-di-GMP concentrations in each strain culture were standardized with the total protein contents determined by Bradford assay [28]. Three biological replicates of each strain were measured and values were expressed as pmol c-di-GMP /mg protein \pm standard error.

\section{Stability of constructs in symbiotic assays}

Bean, vetch and alfalfa seeds (Phaseolus vulgaris cv. Contender, Vicia sativa cv. Jose and Medicago sativa cv. Aragon, respectively) were surface-sterilized and germinated as previously described [13,29]. 12 bean or 25 vetch and alfalfa seedlings were sown in Leonard-type assemblies containing vermiculite:perlite (3:1) in the top part, and nitrogen-free nutrient solution [30] in the bottom. Each seedling was inoculated with $10^{6} \mathrm{CFU}$ of the compatible bacterial symbiont (Ret for beans, Rle for vetch and Sme for alfalfa). Bean and alfalfa plants were cultivated in a growth chamber with 16/8-h light/dark photoperiod at $24 / 16{ }^{\circ} \mathrm{C}$ day/night and $75 \%$ relative humidity. Vetch plants were grown in a greenhouse.

To test the stability in planta of the mini- $\operatorname{Tn} 7$ constructs and plasmids pJB3Tc19 and pJBpleD*, fifty of the nodules formed by each strain were surface-sterilised with $\mathrm{HgCl}_{2} 0.25 \%$ for $5 \mathrm{~min}$ followed by washing with abundant sterile deionised water. Nodules were individually crushed and the content spread on selective (Km or Tc) and nonselective plates. Plasmid stability was determined following the percentage of nodules containing bacteria able to grow in media with antibiotics.

\section{Quantitative RT-PCR assay}

RNA extractions for real-time RT-PCR were carried out using the Qiagen RNeasy RNA purification kit (Qiagen) and following the manufacturer's instructions. Total RNA $(1 \mu \mathrm{g})$ treated with RNase-free Dnase I (Qiagen) was reverse-transcribed using Superscript II reverse transcriptase (Invitrogen) and random hexamers (Roche) as primers. Quantitative real-time PCR was performed with a iCycler iQ5 (Bio-Rad). Each $25 \mu$ reaction contained $1 \mu \mathrm{l} \mathrm{cDNA}, 200 \mathrm{nM}$ of each primer and iQ SyBrGreen Supermix (Bio-Rad). Control PCRs of the RNA 
samples were also performed to confirm the absence of contaminating genomic DNA. Samples were initially denatured by heating at $95^{\circ} \mathrm{C}$ for $3 \mathrm{~min}$, followed by a 35cycle amplification and quantification programme $\left(95^{\circ} \mathrm{C}\right.$ for $30 \mathrm{~s}, 60{ }^{\circ} \mathrm{C}$ for $30 \mathrm{~s}$ and $72{ }^{\circ} \mathrm{C}$ for $30 \mathrm{~s}$ ). Melting curve analysis was conducted to ensure amplification of a single product. The efficiency of each primer pair $(E)$ was determined by running 10-fold serial dilutions (four dilution series) of genomic DNA as template and generating a standard curve by plotting the log of the dilution factor against the $C_{\mathrm{T}}$ value during amplification of each dilution. Amplification efficiency was calculated using the formula $\left[E=\left(10^{(1 / a)}-1\right) \times 100\right]$, where $a$ is the slope of the standard curve. The relative expression of pleD* gene was normalized to that of 16S rRNA gene, which was used as reference.

\section{Construction of a regulatory system of the $p l e D^{*}$ expression in mini-Tn7 strains}

The $\operatorname{lacl}^{q}$ gene was extracted by McsI digestion from the expression vector $\mathrm{pQE}-80 \mathrm{~L}$ (Quiagen). The $1610 \mathrm{bp}$ fragment was cloned into the pBBR1MCS5 [31] previously digested with SmaI. The resulting plasmid $\mathrm{pBBRlacI}^{\mathrm{q}}$ was introduced into Sme Tn7pleD*Km strain using E. coli $\beta 2163$ donor strain as described in [26].

\section{Results and discussion}

Construction and insertion of mini-Tn7 vectors to increase intracellular levels of c-di-GMP in Gram-negative bacteria PleD from Caulobacter crescentus was one of the first and likely the best characterised DGC. It contains two Nterminal receiver domains which regulate DGC activity upon phosphorylation [32]. In contrast, $\mathrm{PleD}^{*}$ is a constitutively active mutant variant of PleD with high DGC activity independent of its phosphorylation status, although it is still subject to feedback regulation through its allosteric I-site, avoiding deleterious effects for uncontrolled DGC activity in the cell $[33,34]$. Plac promoter was selected to control pleD* expression since it behaves as a constitutive promoter in the absence of the LacI repressor. Plac has been used in multiple vectors, including different sets of Tn7 constructs, designed to express a variety of genes in phylogenetically diverse gram-negative and grampositive bacteria, e. g. Pseudomonas aeruginosa, Xanthomonas campestris, Lactobacillus casei, Agrobacterium tumefaciens or Synechococcus elongatus [35-42].

We initially constructed a mini- $\operatorname{Tn} 7$ derivative containing the pleD* gene under the Plac control, miniTn7pleD* which carries no antibiotic selective markers (Fig. 1). This plasmid was maintained in strain E. coli $\beta 2155\left(\right.$ lacl $\left.^{q}\right)$ [25] to prevent undesired PleD* expression in the donor. Triparental matings were performed to deliver the mini-Tn7pleD* plasmid into the genomes of Pseudomonas syringae pv. tomato DC3000 (Pto),
Sinorhizobium meliloti 8530 (Sme), Rhizobium etli CFN42 (Ret) and Rhizobium leguminosarum bv. viciae UPM791 (Rle). E. coli $\beta 2163$ bearing the pUX-BF13 plasmid [17] carrying the transposase genes was used as helper strain for transposition.

ple $D^{*}$ expression in a given strain can be easily followed, as it gives rise to colonies that usually stain red in plates supplemented with Congo Red (CR). We could isolate Ret transposants which formed strong red colonies in CR plates. Such red colonies appeared at a frequency of $10^{-4}$ (Table 1 ) and were easily distinguished amongst hundreds of non-coloured, wild-type colonies. However, the utility of this mini-Tn7pleD* construct without selective markers was limited to bacterial recipients, like $R$. etli, where transposition occurs at a high frequency and transposants display an easily selectable cdi-GMP-dependent phenotype. To facilitate selection of transposants, mini-Tn7pleD*Km and mini-Tn7pleD*Tc vectors, as well as derivatives with a ple ${ }^{*}$ deletion, were constructed (Fig. 1). Transposition efficiencies were relatively low albeit dependent on the bacterial recipient and also on the mini-Tn7 version. In general, transposition efficiencies were higher with $\mathrm{Km}^{\mathrm{r}}$ than with $\mathrm{Tc}^{\mathrm{r}}$ constructs. Furthermore, transposition efficiencies of ple ${ }^{*}$-deleted constructs were generally higher than their $p l e D^{*}$ relatives (Table 1), suggesting that size of the mini-transposon can affect transposition efficiency, as proposed earlier [17].

The location of the mini- $\operatorname{Tn} 7$ insertions in each bacterial strain were determined by PCR and/or Southern hybridization. Pto carries a single copy of $\operatorname{glm} S$ and therefore a single $\operatorname{att} \operatorname{Tn} 7$ site, whereas Rle and Sme genomes have two genes with glucosamine-fructose-6phosphate aminotransferase activity: $g \operatorname{lm} S$ (chromosome) and nodM (Sym plasmid), both associated with att $\operatorname{Tn} 7$ sites. On the other hand, two $\operatorname{glm} S$ genes, $g \operatorname{lm} S 1$ and glmS2 have been annotated in the Ret CFN42 genome [43], however only glmS1 seems to have an att $\operatorname{Tn} 7$ site, according to known att $\operatorname{Tn} 7$ sequences from different bacteria [19, 20, 22, 44, 45].

As expected, in Pto and Ret all transposition events were associated with the $\operatorname{att} \operatorname{Tn} 7$ site located downstream $g \operatorname{lm} S$ and $g \operatorname{lm} S 1$, respectively. In Rle all the transposants analyzed had the mini-Tn 7 insertions downstream the $\operatorname{glm} S$ gene and none were linked to nod $M$. In contrast, in Sme nodM was by far the preferred site of insertion, and only $2 \%$ of the mini-Tn 7 insertions were associated to $g \operatorname{lm} S$, in agreement with previous reports [46]. This is noteworthy, since nodM is part of the nodMnolFGnodN operon and nodM-associated insertions result in undesired polar effects, leading to reduced nodulation efficiency [47, 48]. This site preference, added to the low efficiency of transposition, determined that we could only isolate $\mathrm{g} \operatorname{lm} S$ $\mathrm{Km}^{\mathrm{r}}$, but not $\mathrm{Tc}^{\mathrm{r}}$, transposants in Sme. 


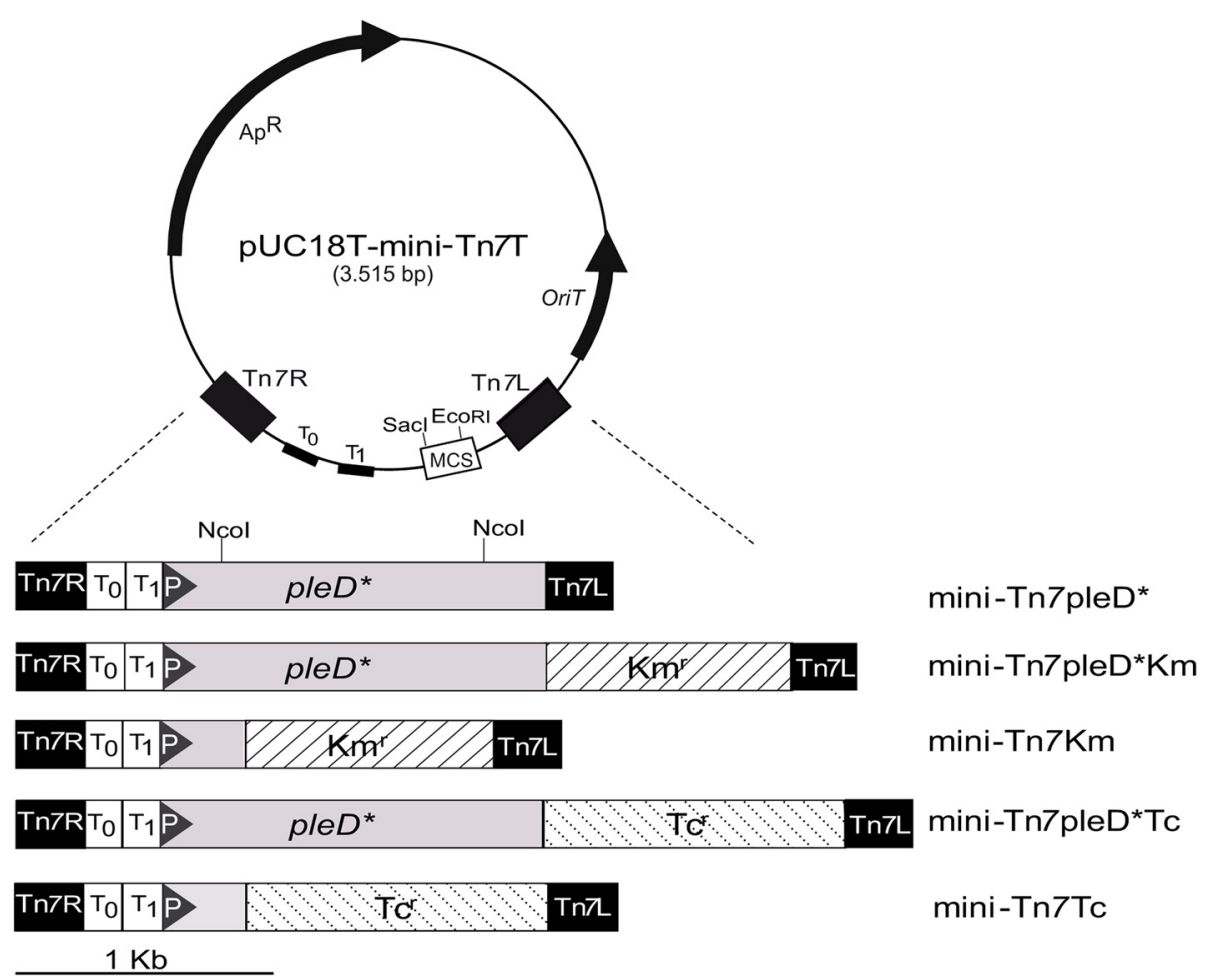

Fig. 1 Mini-Tn7 delivery constructions originated from pUC18T-mini-Tn 7 suicide plasmid. The pleD* gene, under the lac promoter, was cloned between the Tn7 Left and Right ends. Downstream pleD* Tetracycline and Kanamycin resistance genes were cloned to facilitate selection. Vectors with resistance genes but without ple $D^{*}$, were obtained after removing a Ncol internal fragment to $p l e D^{*}$. $A p^{R}, K m^{R}, T c^{R}$ stand for resistance to Ampicillin, Kanamycin and Tetracycline, respectively; MCS, multi-cloning site; $T_{0} T_{1}$, transcriptional terminators from bacteriophage $\lambda$ and $E$. coli rrnB operon, respectively; Tn7L and Tn7R, left and right ends of Tn7 transposon, respectively; P, lac promoter

Table 1 Transposition efficiencies of mini-Tn7 constructs

\begin{tabular}{lll}
\hline Recipient strain & Mini-Tn7 & Frequency $^{a}$ \\
\hline R. etli CFN42 (Ret) & Tn7pleD* & $3,0 \times 10^{-4}$ \\
& Tn7pleD*Km & $6,5 \times 10^{-6}$ \\
& Tn7Km & $5,2 \times 10^{-6}$ \\
& Tn7pleD*Tc & $9,8 \times 10^{-8}$ \\
& Tn7Tc & $5,1 \times 10^{-6}$ \\
R. leguminosarum bv. viciae UPM791 (Rle) & Tn7pleD*Km & $2,5 \times 10^{-8}$ \\
& Tn7Km & $4,8 \times 10^{-8}$ \\
& Tn7pleD*Tc & $<10^{-9}$ \\
& Tn7Tc & $1,8 \times 10^{-9}$ \\
S. meliloti 8530 (Sme) & Tn7pleD*Km & $4 \times 10^{-8}$ \\
& Tn7Km & $8 \times 10^{-8}$ \\
& Tn7pleD*Tc & $3,5 \times 10^{-9}$ \\
& Tn7Tc & $2,3 \times 10^{-8}$ \\
P. syringae pv. tomato DC3000 (Pto) & Tn7pleD*Km & $1,6 \times 10^{-7}$ \\
& Tn7Km & $4,8 \times 10^{-5}$ \\
& Tn7pleD*Tc & $7 \times 10^{-8}$ \\
& Tn7c & $2 \times 10^{-5}$ \\
\hline
\end{tabular}

${ }^{\mathrm{a}} \mathrm{Mini}-\mathrm{Tn} 7$ frequency of transposition expressed as the number of transposants per input receptor cell
c-di-GMP intracellular levels in mini-Tn7pleD* transposants Intracellular c-di-GMP levels of representative miniTn7pleD* transposants were quantified and compared with strains carrying the pJBpleD* plasmid and control strains (Fig. 2). Since $\mathrm{Km}$ and Tc transposants displayed comparable phenotypes (see below), we chose to measure the Tn7pleD*Km transposants as representatives for the rhizobial strains. The levels of wild type rhizobial strains were near the detection limit of the technique used [13]. However, the mini-Tn7pleD*Km transposants showed significantly higher c-di-GMP levels than their respective controls $(\mathrm{Tn} 7 \mathrm{Km})$ in all species, with similar or even higher values than the corresponding derivatives carrying pJBpleD* plasmid (Fig. 2). However, a modest cdi-GMP increment was observed in the Pto Tn7pleD*Km transposant, which showed a three-fold increase above the wild-type levels but three-fold lower levels than the Pto pJBpleD*. In contrast, a Pto Tn7pleD*Tc transposant showed two-fold higher c-di-GMP levels than the Tn7pleD*Km transposant (Fig. 2). The differences between these transposants were probably due to different pleD* expression levels, since the Pto Tn7pleD*Km transposant had 3.7 fold lower pleD* transcripts levels than the Pto Tn7pleD*Tc strain (as measured by qRT- 


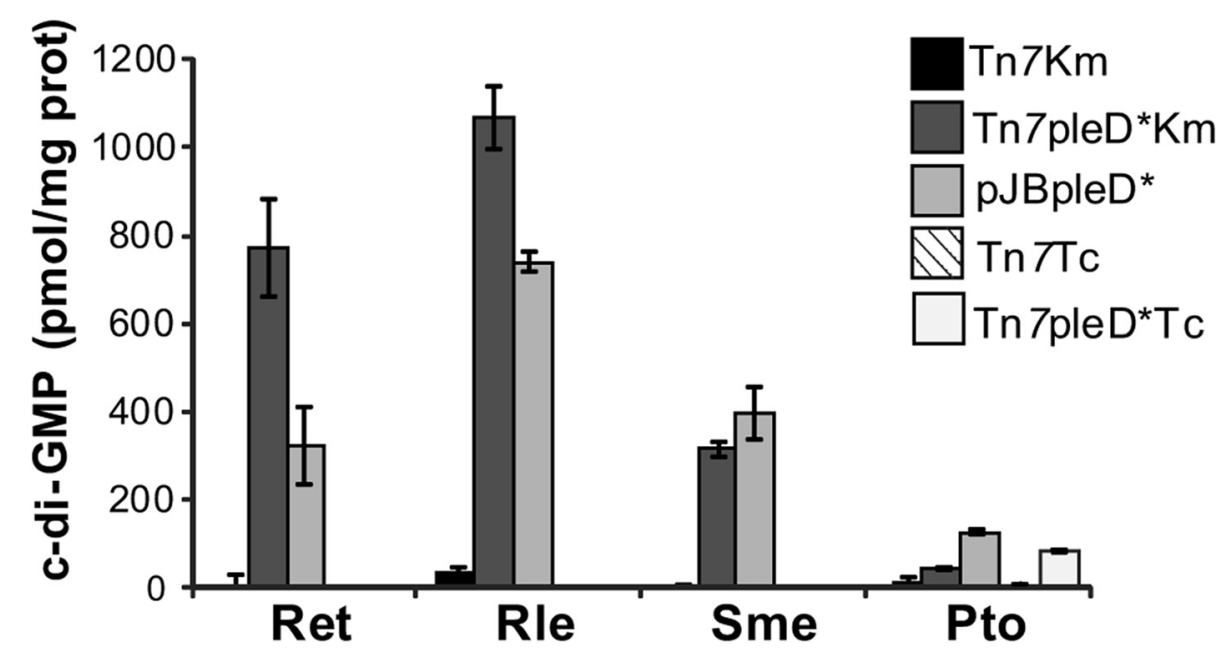

Fig. 2 Quantification of intracellular c-di-GMP levels. c-di-GMP contents of Rhizobium etli CFN42 (Ret), Sinorhizobium meliloti 8530 (Sme), Rhizobium leguminosarum bv. viciae UPM791 (Rle) and Pseudomonas syringae pv. tomato DC3000 (Pto), with plasmid-encoded (pJBpleD*) or chromosomally integrated ple $D^{*}$ gene. Tc transposants in rhizobial strains displayed comparable phenotypes to Km and their c-di-GMP levels were not determined. Control strains without pleD* carried mini-Tn7Km or mini-Tn7Tc. Standard error of three biological replicates are shown

PCR). Although we cannot offer an explanation for these differences, the results suggest the convenience of analyzing several independent transposants with regard to phenotypic changes and c-di-GMP intracellular levels.

Effects of mini-Tn7pleD* insertions on bacterial free-living phenotypes

Raising c-di-GMP levels usually leads to a number of phenotypic changes related to colony morphology, e.g., overproduction of cellulose and other EPSs, and motility reduction in different bacteria [13].
Similar to plasmid pJBpleD*, all mini-Tn7pleD* generated Congo Red $\left(\mathrm{CR}^{+}\right)$and Calcofluor $\left(\mathrm{CF}^{+}\right)$phenotypes (Additional file 1: Figure S1), with an enhanced CFderived fluorescence (Fig. 3). These phenotypes were not observed in non-pleD* strains (Fig. 3 and Additional file 1: Figure S1). CR binds to D-glucopyranosyl units, basic or neutral polysaccharides, as well as to some proteins, whereas CF binds to $\beta(1-4)$ and $\beta(1-3)$ glycosidic bonds of polysaccharides [49]. In Pto, Ret and Rle the $\mathrm{CR}^{+}$and $\mathrm{CF}^{+}$stainings are likely due to overproduction of cellulose [13], whereas in S. meliloti 8530 this is due to another

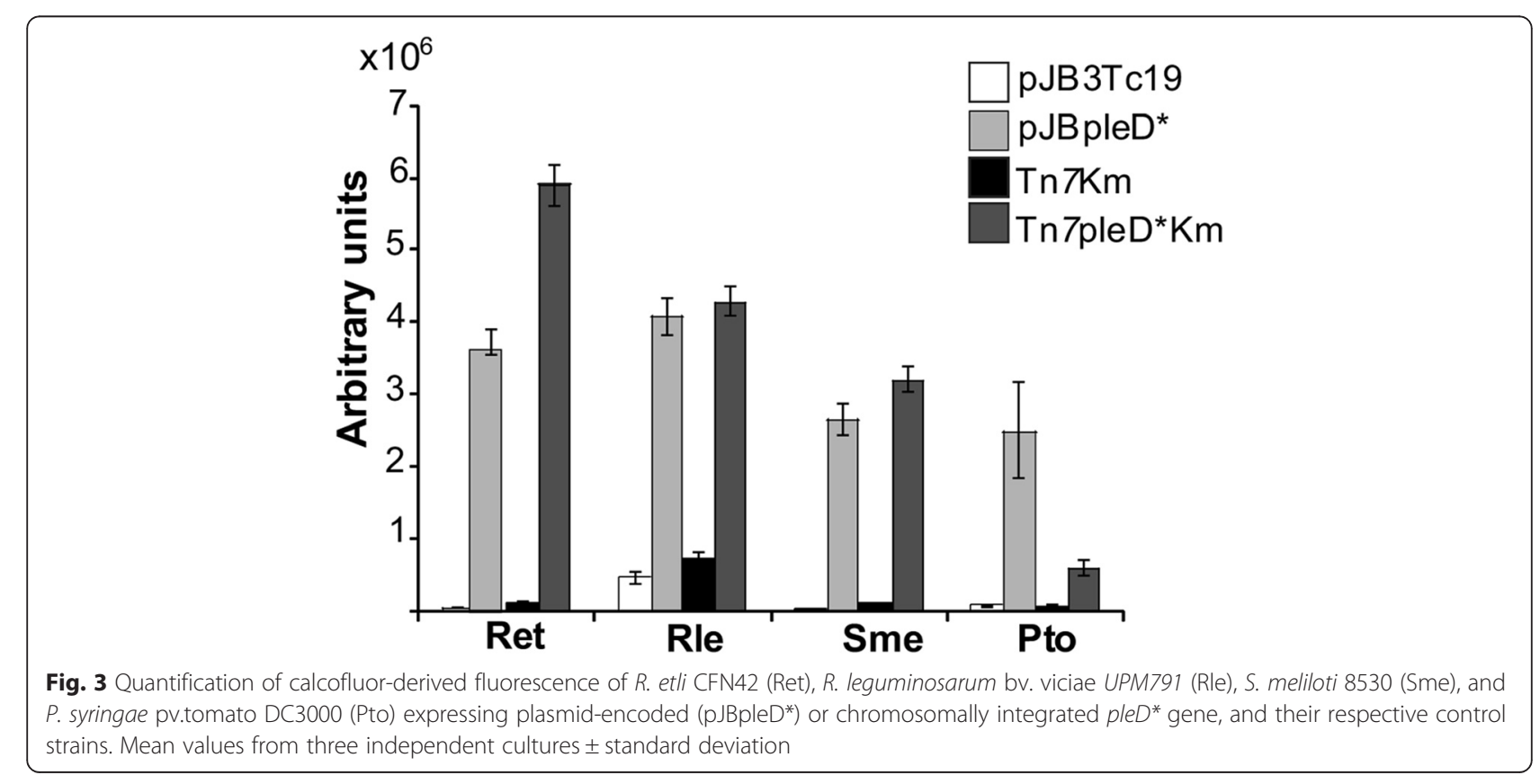


$\mathrm{CR}^{+} / \mathrm{CF}^{+}$related polymer, a recently described mixedlinkage $\beta$-glucan [15]. Stronger CR and CF stainings were observed for the Pto Tn7pleD*Tc than for the Pto Tn7pleD"Km strain, in agreement with the higher c-diGMP intracellular and ple $D^{*}$ expression levels in that transposant, as described above (Fig. 2 and Additional file 1: Figure S1).

Correlating with enhanced polysaccharide production, all strains expressing $p l e D^{*}$ showed a strong aggregative behaviour, forming flocs in liquid media (data not shown). Enhanced polysaccharide production usually leads to biofilm formation [50-52]. The ple $D^{*}$ expression, either from plasmid or from integrated miniTn7pleD* constructs, similarly led to an enhanced biofilm formation, which was quantified in Ret and Rle by Cristal Violet staining in microtiter plates (Additional file 1: Figure S2). Pto and Sme, on the other hand, formed air-liquid interface biofilms, pellicles that easily collapsed and could not be retained during the $\mathrm{CV}$ staining procedures, hindering quantification [13].

High c-di-GMP levels usually inhibit bacterial motility [53-57]. As expected, mini-Tn7pleD* transposants displayed strongly reduced swarming and swimming motilities in all strains (Additional file 1: Figure S3), in a similar way to what has been reported for pJBpleD*carrying bacteria [13].

\section{Stability of mini-Tn7pleD* insertions}

Stability of mini-Tn7 insertions was determined after 100 generations free-living growth in rich broth without antibiotic selective pressure and compared with the plasmid pJBpleD* or the empty vector pJB3Tc19. As reported previously [13] the pJB3Tc19 and especially the pJBpleD* plasmids were rapidly lost in the absence of Tc, particularly in Ret and Sme with less than 0.6 and $3.1 \%$, respectively, of $\mathrm{Tc}^{\mathrm{r}}$ CFUs retaining pJBpleD* after 100 generations (Table 2). In stark contrast, all mini-Tn7 insertions were $100 \%$ stable in all strains tested. The instability of pleD" plasmid under non selective pressure could also be observed for free-living phenotypes (Additional file 1: Figure S4). For instance, strain Ret pJBpleD* under non selective conditions showed a progressive loss of the CR binding capacity, which gave rise to a segmented-colony phenotype after 5 days of growth, with most cells at the colony edges showing a $\mathrm{CR}^{-}$phenotype (Additional file 1: Figure S4b). This was not observed with this strain in the presence of Tc. In contrast, the transposant Ret Tn7pleD*Tc strain did not show the colony sectored appearance, either in the presence or in the absence of Tc (Additional file 1: Figure S4b). Likewise, Ret Tn7pleD*Tc strain displayed a complete arrest of swimming motility either in the presence or absence
Table 2 Stability of plasmids and mini-Tn7 insertions in different strains

\begin{tabular}{|c|c|c|}
\hline Strain & Free-living stability ${ }^{a}$ & Stability in symbiosis \\
\hline Ret pJB3Tc19 & $8,6 \%$ & $98 \%$ \\
\hline Ret pJBpleD* & $0.6 \%$ & $0 \%$ \\
\hline Ret Tn7Km & $100 \%$ & $100 \%$ \\
\hline Ret Tn7pleD*Km & $100 \%$ & $90 \%$ \\
\hline Ret $T n 7 T c$ & $100 \%$ & n.d. \\
\hline Ret Tn7pleD*Tc & $100 \%$ & n.d. \\
\hline Rle pJB3Tc19 & $73,5 \%$ & $86 \%$ \\
\hline Rle pJBpleD* & $67,1 \%$ & $70 \%$ \\
\hline Rle Tn7Km & $100 \%$ & $100 \%$ \\
\hline RleTn7pleD*Km & $100 \%$ & $100 \%$ \\
\hline Rle $T n \pi T c$ & $100 \%$ & n.d. \\
\hline Rle Tn7pleD*Tc & n.d & n.d. \\
\hline Sme pJB3Tc19 & $2,8 \%$ & $51 \%$ \\
\hline Sme pJBpleD* & $3,1 \%$ & $22 \%$ \\
\hline Sme Tn7Km & $100 \%$ & $100 \%$ \\
\hline Sme Tn7pleD*Km & $100 \%$ & $91 \%$ \\
\hline Sme Tn $7 \mathrm{c}$ & $100 \%$ & n.d. \\
\hline Sme Tn7pleD*Tc & $100 \%$ & n.d. \\
\hline Pto pJB3Tc19 & $100 \%$ & n.d. \\
\hline Pto pJBpleD* & $76,8 \%$ & n.d. \\
\hline Pto Tn7Km & $100 \%$ & n.d. \\
\hline Pto Tn7pleD*Km & $100 \%$ & n.d. \\
\hline Pto $T n \pi T c$ & $100 \%$ & n.d. \\
\hline Pto Tn7pleD*Tc & $100 \%$ & n.d. \\
\hline
\end{tabular}

${ }^{\mathrm{a} B a c t e r i a}$ were grown in rich medium without antibiotics for at least 100 generations; the stability was determined as the ratio of CFU recovered on selective medium out of the total CFU obtained in nonselective medium bercentage of nodules containing bacteria that kept antibiotic resistance (for more details see supporting information)

n.d., not determined

of tetracycline, whereas strain Ret pJBpleD* showed reduced (but not fully arrested) swimming, and formed swimming haloes which were significantly larger in media without antibiotic pressure, evidencing loss of the pJBpleD* plasmid (Additional file 1: Figure S4a).

The stability of mini-Tn7 constructs in different rhizobial strains were also evaluated in planta (Table 2). Bacteria which kept the Tn7pleD*Km could be recovered from 90 to $100 \%$ of root nodules. In contrast, the maintenance of pJBPleD* plasmid in nodules was significantly lower, being negligible or even undetectable in some cases (Table 2). This emphasizes the utility of our mini-Tn7pleD* constructs under experimental conditions where antibiotic selection is not feasible. Nevertheless, stability of miniTn7pleD*Km insertions in Sme and Ret seemed slightly lower in planta (90\%) than in free-living conditions (100\%; Table 2), indicating that during nodule infection 
Table 3 Efficacy of the lacl ${ }^{9}-$ IPTG system to modulate pleD* expression

\begin{tabular}{|c|c|c|c|c|c|c|}
\hline \multirow[t]{2}{*}{ Strain } & \multicolumn{2}{|c|}{ Relative pleD* expression ${ }^{1}$} & \multicolumn{2}{|c|}{ Intracellular c-di-GMP ${ }^{2}$} & \multicolumn{2}{|l|}{ EPS production ${ }^{3}$} \\
\hline & IPTG - & IPTG + & IPTG - & IPTG + & IPTG - & IPTG + \\
\hline Sme Tn7pleD*Km pBBR1MCS5 & $15,11 \pm 1,98$ & n.d. & $317,16 \pm 13,29$ & n.d. & $2,66 \times 10^{6} \pm 6,9 \times 10^{4}$ & $2,64 \times 10^{6} \pm 7,34 \times 10^{4}$ \\
\hline Sme Tn7pleD*Km pBBRlacl ${ }^{q}$ & $1,00 \pm 0,26$ & $6,08 \pm 1,06$ & $55,10 \pm 16,27$ & $152,05 \pm 8,50$ & $3,51 \times 10^{5} \pm 1,19 \times 10^{4}$ & $2,23 \times 10^{6} \pm 6,36 \times 10^{4}$ \\
\hline Sme Tn7Km & - & - & - & n.d. & $6,20 \times 10^{4} \pm 5,54 \times 10^{3}$ & $7,39 \times 10^{4} \pm 3,44 \times 10^{3}$ \\
\hline
\end{tabular}

${ }^{1}$ Relative expression (fold change) to Sme Tn7pleD*Km pBBRlacl ${ }^{q}$ strain without IPTG (repression state) by qRT-PCR. In all strains pleD* expression was normalized with to $16 \mathrm{~S}$ rRNA levels

${ }^{2} \mathrm{pmol}$ of c-di-GMP/mg of total protein. The c-di-GMP levels of Sme Tn7Km is under the technical limit of detection

${ }^{3} \mathrm{CF}$-derived Fluorescence (arbitrary units)

n.d., not determined

there is a strong pressure against bacteria expressing high c-di-GMP levels, as suggested earlier [13].

\section{Modulation of pleD* expression in mini-Tn7 strains}

Overexpression of DGCs usually have a deep impact in the c-di-GMP economy, generating strong phenotypes [58-60]. In that sense, modulating DGC expression in these mini-Tn7pleD* transposants could be useful in order to raise the intracellular levels of c-di-GMP “on-demand”. Since pleD* transcription is under the control of lac promoter, we evaluated if ple ${ }^{*}$ expression could be modulated in these miniTn7 transposants using a $l a c I^{q}$-based system and the inducer Isopropyl $\beta$-D-1-thiogalactopyranoside (IPTG). In order to keep the $p l e D^{*}$ expression at a minimum under non inducible conditions, we cloned the repressor $\operatorname{lacl}^{q}$ gene version from $\mathrm{pQE}-80 \mathrm{~L}$ into the broad host range plasmid pBBR1MCS-5 [31], obtaining pBBRlacI ${ }^{\mathrm{q}}$. This plasmid was introduced by conjugation in the Sme Tn7pleD*Km strain. pleD* expression, c-di-GMP levels and EPS production were determined in the absence and in the presence of IPTG (Table 3). qRT-PCR confirmed that pleD* was strongly repressed in the presence of LacI (Tn7pleD*Km pBBRlacI ${ }^{\mathrm{q}}$ ) and absence of inducer. This repression state was alleviated by $1 \mathrm{mM}$ IPTG. However, even with this high amounts of inducer, pleD* transcription did not reach the levels attained in the absence of the LacI repressor. The intracellular levels of c-di-GMP followed the same trend (Table 3). $1 \mathrm{mM}$ of IPTG generated a 3-fold increase of c-di-GMP levels in strain Sme Tn7pleD*Km pBBRlacI $^{q}$, just half of the rise achieved in the absence of the repressor (Sme Tn7pleD*Km pBBR1MCS5). However, this addition of IPTG was enough to produce similar impacts on EPS production, increasing it up to 1 Log (Table 3 and Additional file 1: Figure S5), indicating that maximum EPS production can be achieved at intermediate c-diGMP levels. Overall these results demonstrate that the $l a c I^{\mathrm{q}} / \mathrm{IPTG}$ system could be useful to modulate the c-di-GMP intracellular levels generated by the mini-Tn7pleD* insertions. Even when a complete derepression of pleD* by IPTG could not be achieved,
c-di-GMP levels were sufficiently high to generate the expected phenotypes.

\section{Conclusions}

In this work, we have constructed a set of mini-Tn7 vehicles to allow integration and stable expression of a DGC gene, ple ${ }^{*}$, useful to artificially increase the intracellular levels of the second messenger c-di-GMP in bacteria. The utility of these new mini-Tn7pleD* tools has been proved in several $\alpha$ - and $\gamma$-proteobacteria, where significant increases in intracellular c-di-GMP contents are achieved. As a consequence, phenotypic changes such as enhanced polysaccharide production and biofilm formation, and reduced motility are easily observed. Nonetheless, a careful interpretation of the bacterial phenotypes generated by the high non-physiological ple ${ }^{*}$-dependent c-di-GMP levels should be exercised. The highly stable mini-Tn7pleD* constructs are particularly convenient under conditions where a selective pressure cannot be applied to ensure DGC expression, e.g., during interaction with an eukaryotic host. Furthermore, we have also implemented an inducible system to modulate pleD $^{*}$ expression and intracellular c-di-GMP rises on demand.

\section{Additional file}

\begin{abstract}
Additional file 1: Table S1. Bacterial strains and plasmids used in this work. Table S2. Primers used in this work. Figure S1. Congo red (CR) and calcofluor (CF) staining of R. etli CFN42 (Ret), S. meliloti 8530 (Sme), R. leguminosarum bv. viciae UPM791 (Rle) and P. syringae pv.tomato DC3000 (Pto) expressing pleD* in mono and multicopy and their respectives control strains. Figure S2. Biofilm formation by $R$. etli CFN42 (Ret) and $R$. leguminosarum bv. viciae UPM791 (Rle) strains expressing pleD* in

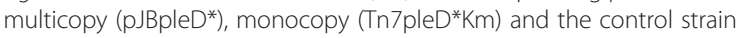
without pleD* $(\mathrm{Tn} 7 \mathrm{Km})$. Figure $\mathbf{S 3}$. Motility reduction in mini-Tn7pleD* strains. Figure S4. Stability of mini-Tn7pleD* in R. etli CFN42 (Ret) under non selective conditions. Figure S5. Control expression of pleD* by $l a l^{q} /$ IPTG system. Colony morphology of S. meliloti (Sme) after grown for two days on MM plates supplemented with Congo Red (CR) with and without the inducer IPTG (1 mM). (PDF $2631 \mathrm{~kb})$
\end{abstract}

\section{Competing interests}

The authors declare that they have no competing interests. 


\section{Authors' contributions}

LRJ and DRC carried out the experimental procedures, processed data and have been involved in drafting the manuscript. MTG, JSP and DPM participated in experimental design, data management and discussion, and helped to manuscript draft. DPM conceived and coordinated the study and the writing of the manuscript. All authors read and approved the final manuscript.

\section{Authors' information}

Not applicable.

\section{Acknowledgments}

This work was supported by grants BIO2011-23032 and BIO2014-55075-P (Ministerio de Economía y Competitividad) and P10-CVI-5800 (Junta de Andalucía), all co-financed with FEDER funds, and CSIC 201440E026. LRJ was supported by JAE-Pre fellowship, and DRC by a contract associated to BIO2011-23032. DPM was supported by a JAE-Doc grant and contracts associated to grants P10-CVI-5800 and CSIC 201440E026.

\section{Received: 17 July 2015 Accepted: 18 September 2015}

Published online: 29 September 2015

\section{References}

1. Römling U, Galperin MY, Gomelsky M. Cyclic di-GMP: the first 25 years of a universal bacterial second messenger. Microbiol Mol Biol Rev. 2013;77(1):1-52

2. Mills E, Pultz IS, Kulasekara HD, Miller SI. The bacterial second messenger c-di-GMP: mechanisms of signalling. Cell Microbiol. 2011;13(8):1122-9.

3. Sondermann $\mathrm{H}$, Shikuma NJ, Yildiz FH. You've come a long way: c-di-GMP signaling. Curr Opin Microbiol. 2012;15(2):140-6.

4. Römling U, Gomelsky M, Galperin MY. C-di-GMP: the dawning of a novel bacterial signalling system. Mol Microbiol. 2005;57(3):629-39.

5. Galperin MY, Koonin EV. From complete genome sequence to 'complete' understanding? Trends Biotechnol. 2010;28(8):398-406.

6. Amikam D, Galperin MY. PilZ domain is part of the bacterial c-di-GMP binding protein. Bioinformatics. 2006;22(1):3-6.

7. Fang X, Ahmad I, Blanka A, Schottkowski M, Cimdins A, Galperin MY, et al. GIL, a new c-di-GMP-binding protein domain involved in regulation of cellulose synthesis in enterobacteria. Mol Microbiol. 2014;93(3):439-52.

8. Ryjenkov DA, Simm R, Römling U, Gomelsky M. The PilZ domain is a receptor for the second messenger c-di-GMP: the PilZ domain protein YcgR controls motility in enterobacteria. J Biol Chem. 2006;281(41):30310-4.

9. Tschowri N, Schumacher MA, Schlimpert S, Chinnam NB, Findlay KC, Brennan RG, et al. Tetrameric c-di-GMP mediates effective transcription factor dimerization to control Streptomyces development. Cell. 2014;158(5):1136-47.

10. Monds RD, Newell PD, Gross RH, O'Toole GA. Phosphate-dependent modulation of c-di-GMP levels regulates Pseudomonas fluorescens PfO- 1 biofilm formation by controlling secretion of the adhesin LapA. Mol Microbiol. 2007;63(3):656-79.

11. Wolfe AJ, Visick KL. Get the message out: cyclic-Di-GMP regulates multiple levels of flagellum-based motility. J Bacteriol. 2008;190(2):463-75.

12. Zorraquino V, García B, Latasa C, Echeverz M, Toledo-Arana A, Valle J, et al. Coordinated cyclic-di-GMP repression of Salmonella motility through YcgR and cellulose. J Bacteriol. 2013;195(3):417-28.

13. Pérez-Mendoza D, Aragon IM, Prada-Ramírez HA, Romero-Jiménez L, Ramos C, Gallegos MT, et al. Responses to elevated c-di-GMP levels in mutualistic and pathogenic plant-interacting bacteria. PLoS One. 2014;9(3), e91645.

14. Pérez-Mendoza D, Coulthurst SJ, Sanjuán J, Salmond GP. N-acetylglucosamine-dependent biofilm formation in Pectobacterium atrosepticum is cryptic and activated by elevated c-di-GMP levels. Microbiology. 2011;157(12):3340-8.

15. Pérez-Mendoza D, Rodríguez-Carvajal MA, Romero-Jiménez L, Farias Gde A Lloret J, Gallegos MT, et al. Novel mixed-linkage beta-glucan activated by c-di-GMP in Sinorhizobium meliloti. Proc Natl Acad Sci U S A. 2015;112(7):E757-65

16. Choi KH, Gaynor JB, White KG, Lopez C, Bosio CM, Karkhoff-Schweizer RR, et al. A Tn7-based broad-range bacterial cloning and expression system. Nat Methods. 2005;2(6):443-8.
17. Bao Y, Lies DP, Fu H, Roberts GP. An improved Tn7-based system for the single-copy insertion of cloned genes into chromosomes of gram-negative bacteria. Gene. 1991;109(1):167-8

18. Peters JE, Craig NL. Tn7: smarter than we thought. Nat Rev Mol Cell Biol. 2001;2(11):806-14.

19. Waddell CS, Craig NL. Tn7 transposition: recognition of the attTn7 target sequence. Proc Natl Acad Sci U S A. 1989;86(11):3958-62.

20. Choi KH, Deshazer D, Schweizer HP. mini-Tn7 insertion in bacteria with multiple glmS-linked attTn7 sites: example Burkholderia mallei ATCC 23344 Nat Protoc. 2006;1(1):162-9.

21. Choi KH, Schweizer HP. mini-Tn7 insertion in bacteria with secondary, non-glmS-linked attTn7 sites: example Proteus mirabilis HI4320. Nat Protoc. 2006;1(1):170-8.

22. Choi $\mathrm{KH}$, Schweizer HP. mini-Tn7 insertion in bacteria with single attTn7 sites: example Pseudomonas aeruginosa. Nat Protoc. 2006;1(1):153-61.

23. Beringer JE. R factor transfer in Rhizobium leguminosarum. J Gen Microbiol. 1974:84(1):188-98.

24. Robertsen BK, Aman P, Darvill AG, McNeil M, Albersheim P. The structure of acidic extracellular polysaccharides secreted by Rhizobium leguminosarum and Rhizobium trifolii. Plant Physiol. 1981;67(3):389-400.

25. Demarre G, Guerout AM, Matsumoto-Mashimo C, Rowe-Magnus DA Marliere P, Mazel D. A new family of mobilizable suicide plasmids based on broad host range R388 plasmid (IncW) and RP4 plasmid (IncPalpha) conjugative machineries and their cognate Escherichia coli host strains. Res Microbiol. 2005;156(2):245-55.

26. Pérez-Mendoza D, Sepúlveda E, Pando V, Muñoz S, Nogales J, Olivares J, et al. Identification of the rctA gene, which is required for repression of conjugative transfer of rhizobial symbiotic megaplasmids. J Bacteriol. 2005:187(21):7341-50.

27. Soto MJ, Fernández-Pascual M, Sanjuán J, Olivares J. A fadD mutant of Sinorhizobium meliloti shows multicellular swarming migration and is impaired in nodulation efficiency on alfalfa roots. Mol Microbiol. 2002;43(2):371-82.

28. Bradford MM. A rapid and sensitive method for the quantitation of microgram quantities of protein utilizing the principle of protein-dye binding. Anal Biochem. 1976;72:248-54.

29. Olivares J, Casadesús J, Bedmar EJ. Method for testing degree of infectivity of Rhizobium meliloti strains. Appl Environ Microbiol. 1980;39(5):967-70.

30. Rigaud J, Puppo A. Indole-3-acetic acid catabolism by soybean bacteroids. J Gen Microbiol. 1975:88(2):223-8.

31. Kovach ME, Elzer PH, Hill DS, Robertson GT, Farris MA, Roop RM, et al. Four new derivatives of the broad-host-range cloning vector PBBR1MCS, carrying different antibiotic-resistance cassettes. Gene. 1995;166(1):175-6.

32. Paul R, Weiser S, Amiot NC, Chan C, Schirmer T, Giese B, et al. Cell cycledependent dynamic localization of a bacterial response regulator with a novel di-guanylate cyclase output domain. Genes Dev. 2004;18(6):715-27.

33. Aldridge $P$, Paul $R$, Goymer $P$, Rainey $P$, Jenal U. Role of the GGDEF regulator PleD in polar development of Caulobacter crescentus. Mol Microbiol. 2003;47(6):1695-708.

34. Jenal U, Malone J. Mechanisms of cyclic-di-GMP signaling in bacteria. Annu Rev Genet. 2006;40:385-407.

35. Chen CY, Winans SC. Controlled expression of the transcriptional activator gene virG in Agrobacterium tumefaciens by using the Escherichia coli lac promoter. J Bacteriol. 1991;173(3):1139-44.

36. Jaenecke S, Díaz E. Construction of plasmid vectors bearing a Notl-expression cassette based on the lac promoter. Int Microbiol. 1999;2(1):29-31.

37. Koch $B$, Jensen LE, Nybroe O. A panel of Tn7-based vectors for insertion of the gfp marker gene or for delivery of cloned DNA into Gram-negative bacteria at a neutral chromosomal site. J Microbiol Methods. 2001;45(3):187-95

38. Lambertsen L, Sternberg C, Molin S. Mini-Tn7 transposons for site-specific tagging of bacteria with fluorescent proteins. Environ Microbiol. 2004;6(7):726-32

39. Noriega CE, Sharma V, Rowe JJ. Artificial control of nitrate respiration through the lac promoter permits the assessment of oxygen-mediated posttranslational regulation of the nar operon in Pseudomonas aeruginosa. J Bacteriol. 2007;189(17):6501-5.

40. Nozzi NE, Atsumi S. Genome Engineering of the 2,3-Butanediol Biosynthetic Pathway for Tight Regulation in Cyanobacteria. ACS Synth Biol. 2015. doi:10.1021/acssynbio.5b00057. 
41. Pérez-Arellano I, Pérez-Martinez G. Structural features of the lac promoter affecting gusA expression in Lactobacillus casei. Curr Microbiol. 2002;45(3):191-6

42. Soby SD, Daniels MJ. Catabolite-repressor-like protein regulates the expression of a gene under the control of the Escherichia coli lac promoter in the plant pathogen Xanthomonas campestris pv. campestris. Appl Microbiol Biotechnol. 1996;46(5-6):559-61.

43. González V, Santamaria RI, Bustos P, Hernández-Gonzalez I, Medrano-Soto A, Moreno-Hagelsieb G, et al. The partitioned Rhizobium etli genome: genetic and metabolic redundancy in seven interacting replicons. Proc Natl Acad Sci USA. 2006;103(10):3834-9.

44. Jittawuttipoka T, Buranajitpakorn S, Fuangthong M, Schweizer HP, Vattanaviboon P, Mongkolsuk S. Mini-Tn7 vectors as genetic tools for gene cloning at a single copy number in an industrially important and phytopathogenic bacteria, Xanthomonas spp. FEMS MicrobiolLett. 2009;298(1):111-7.

45. Kumar A, Dalton C, Cortez-Cordova J, Schweizer HP. Mini-Tn7 vectors as genetic tools for single copy gene cloning in Acinetobacter baumannii. J Microbiol Methods. 2010;82(3):296-300,

46. Bolton E, Glynn P, O'Gara F. Site specific transposition of Tn7 into a Rhizobium meliloti megaplasmid. Molec Gen Genet. 1984;193(1):153-7.

47. Baev N, Endre G, Petrovics G, Banfalvi Z, Kondorosi A. Six nodulation genes of nod box locus 4 in Rhizobium meliloti are involved in nodulation signal production: nodM codes for D-glucosamine synthetase. Mol Gen Genet. 1991;228(1-2):113-24.

48. Baev N, Schultze M, Barlier I, Ha DC, Virelizier H, Kondorosi E, et al. Rhizobium nodM and nodN genes are common nod genes: nodM encodes functions for efficiency of nod signal production and bacteroid maturation. J Bacteriol. 1992;174(23):7555-65.

49. Spiers AJ, Kahn SG, Bohannon J, Travisano M, Rainey PB. Adaptive divergence in experimental populations of Pseudomonas fluorescens. I. Genetic and phenotypic bases of wrinkly spreader fitness. Genetics. 2002;161(1):33-46.

50. Danhorn T, Fuqua C. Biofilm formation by plant-associated bacteria. Annu Rev Microbiol. 2007:61:401-22.

51. OToole GA, Pratt LA, Watnick PI, Newman DK, Weaver VB, Kolter R. Genetic approaches to study of biofilms. Methods Enzymol. 1999:310:91-109.

52. Rinaudi LV, Giordano W. An integrated view of biofilm formation in rhizobia. FEMS MicrobiolLett. 2010;304(1):1-11.

53. Kuchma SL, Brothers KM, Merritt JH, Liberati NT, Ausubel FM, OToole GA BifA, a cyclic-Di-GMP phosphodiesterase, inversely regulates biofilm formation and swarming motility by Pseudomonas aeruginosa PA14. J Bacteriol. 2007;189(22):8165-78.

54. Pérez-Mendoza D, Coulthurst SJ, Humphris S, Campbell E, Welch M, Toth IK, et al. A multi-repeat adhesin of the phytopathogen, Pectobacterium atrosepticum, is secreted by a Type I pathway and is subject to complex regulation involving a non-canonical diguanylate cyclase. Mol Microbiol. 2011:82(3):719-33.

55. Baraquet C, Harwood CS. Cyclic diguanosine monophosphate represses bacterial flagella synthesis by interacting with the Walker A motif of the enhancer-binding protein FleQ. Proc Natl Acad Sci U S A. 2013;110(46):18478-83.

56. D'Argenio DA, Miller SI. Cyclic di-GMP as a bacterial second messenger. Microbiology. 2004;150(Pt 8):2497-502

57. Hengge R. Principles of c-di-GMP signalling in bacteria. Nat Rev Microbiol. 2009;7(4):263-73.

58. Edmunds AC, Castiblanco LF, Sundin GW, Waters CM. Cyclic Di-GMP modulates the disease progression of Erwinia amylovora. J Bacteriol. 2013;195(10):2155-65.

59. Simm R, Morr M, Kader A, Nimtz M, Römling U. GGDEF and EAL domains inversely regulate cyclic di-GMP levels and transition from sessility to motility. Mol Microbiol. 2004;53(4):1123-34

60. Yan H, Chen W. 3',5'-Cyclic diguanylic acid: a small nucleotide that makes big impacts. Chem Soc Rev. 2010;39(8):2914-24.

\section{Submit your next manuscript to BioMed Central and take full advantage of:}

- Convenient online submission

- Thorough peer review

- No space constraints or color figure charges

- Immediate publication on acceptance

- Inclusion in PubMed, CAS, Scopus and Google Scholar

- Research which is freely available for redistribution

Submit your manuscript at www.biomedcentral.com/submit 